\title{
Relation between the kinetics of thallium-201 in myocardial scintigraphy and myocardial metabolism in patients with acute myocardial infarction
}

H Yamagishi, K Akioka, M Takagi, A Tanaka, K Takeuchi, J Yoshikawa, H Ochi

\begin{abstract}
Objective-To investigate the relations between myocardial metabolism and the kinetics of thallium-201 in myocardial scintigraphy.

Methods-46 patients within six weeks after the onset of acute myocardial infarction underwent resting myocardial dual isotope, single acquisition, single photon emission computed tomography (SPECT) using radioiodinated 15-iodophenyl 3-methyl pentadecaenoic acid (BMIPP) and thallium-201, exercise thallium-201 SPECT, and positron emission tomography (PET) using nitrogen-13 ammonia $\left(\mathrm{NH}_{3}\right)$ and [F18]fluorodeoxyglucose (FDG) under fasting conditions. The left ventricle was divided into nine segments, and the severity of defects was assessed visually.

Results-In the resting SPECT, less BMIPP uptake than thallium-201 uptake was observed in all of 40 segments with reverse redistribution of thallium-201, and in 21 of 88 segments with a fixed defect of thallium-201 ( $p<0.0001)$; and more FDG uptake than $\mathrm{NH}_{3}$ uptake $\left(\mathrm{NH}_{3}-\mathrm{FDG}\right.$ mismatch) was observed in 35 of 40 segments with reverse redistribution and in 38 of 88 segments with fixed defect $(p<0.0001)$. Less BMIPP uptake in the resting SPECT was observed in 49 of 54 segments with slow stress redistribution in exercise SPECT, and in nine of 17 segments with rapid stress redistribution $(\mathrm{p}<0.0005)$; $\mathrm{NH}_{3}-$ FDG mismatch was observed in 42 of 54 segments with slow stress redistribution and in five of 17 segments with rapid stress redistribution $(\mathbf{p}<0.0005)$.

Conclusions-Thallium-201 myocardial scintigraphy provides information about not only myocardial perfusion and viability but also about myocardial metabolism in patients with acute myocardial infarction.

(Heart 1998;80:28-34)
\end{abstract}

Keywords: thallium-201 SPECT; BMIPP SPECT; FDG PET; myocardial infarction; redistribution

In thallium-201 myocardial scintigraphy, perfusion defects in images obtained immediately after exercise (initial image) may be no longer evident in the images obtained several hours after exercise (delayed image). ${ }^{1}$ This phenomenon is referred to as redistribution, and is thought to indicate the presence of myocardial ischaemia.

Reverse redistribution refers to perfusion defects that appear, or become more evident, in the delayed images obtained in resting or stress thallium-201 myocardial scans, ${ }^{2}$ and is very common in patients with acute myocardial infarction treated with thrombolysis. ${ }^{3}$ Although the significance and the mechanism of this phenomenon remain controversial, ${ }^{2}$ we have reported that reverse redistribution on resting myocardial scintigraphy in patients with acute myocardial infarction is observed in viable myocardium with severe contraction abnormality, ${ }^{4}$ and is closely related to the slow stress redistribution observed in four hour delayed images in exercise scintigraphy. ${ }^{5}$

In many regions of the viable myocardium, perfusion defects that are detected on the initial images persist, and appear to be irreversible, on delayed images obtained three to four hours after exercise. ${ }^{6}$ Some persistent defects appear to have improved in the delayed images obtained eight to 72 hours after thallium-201 injection, ${ }^{78}$ in images obtained after reinjection of additional thallium-201, ${ }^{10}$ or when a resting study is repeated with an additional injection of thallium-201. ${ }^{112}$ Thus it is widely accepted that perfusion defects observed in the delayed image obtained three to four hours after exercise underestimates the viability of myocardium in the infarct region, and that the redistribution in the delayed image obtained three to four hours after exercise is insufficient.

Myocardial metabolic imaging with the use of fatty acid analogues and glucose analogues has recently become available. Radioiodinated 15-iodophenyl 3-methyl pentadecaenoic acid (BMIPP) is used clinically as a probe for studying the myocardial metabolism of fatty acids. ${ }^{14}$ Positron emission tomography (PET) allows assessment of regional myocardial perfusion and metabolism in vivo. Hypoperfused myocardium with an increased uptake of [F18]fluorodeoxyglucose (FDG) as an indicator of exogenous glucose utilisation is considered to be an ischaemic but viable myocardium with potential for improvement in regional function after the restoration of blood flow. ${ }^{15} 16$

Although Matsunari et al have reported that fatty acid uptake is impaired in the area of fill-in after reinjection on exercise thallium-201 scintigraphy, ${ }^{17}$ the relation between myocardial metabolism and the kinetics of thallium-201 
remains to be clarified. Our aim in this study was to measure the uptake of fatty acids and glucose in the myocardium, demonstrating reverse redistribution in resting thallium-201 myocardial scintigraphy or slow stress redistribution on the four hour delayed image from exercise thallium-201 scintigraphy. Uptake was measured using BMIPP single photon emission computed tomography (SPECT) and FDG PET.

\section{Methods}

PATIENT POPULATION

Forty six patients with acute myocardial infarction (39 men and seven women; mean (SD) age 60 (10) years, range 33 to 74 years) who were admitted to the coronary care unit of Osaka City University Medical School Hospital between October 1993 and June 1996 and underwent resting myocardial dual isotope, single acquisition SPECT using BMIPP and thallium-201, exercise thallium-201 SPECT and PET using FDG and nitrogen-13 ammonia $\left(\mathrm{NH}_{3}\right)$ in the subacute phase (within six weeks after the onset of myocardial infarction) were selected for the present study retrospectively from our patient database. Acute myocardial infarction was defined by the presence of typical chest pain, depression or elevation of the ST segment on standard 12 lead electrocardiogram, and a rise in serum creatine kinase or the creatine kinase $\mathrm{MB}$ isoenzyme to more than three times the upper limit of normal. Patients with a history of previous myocardial infarction or of coronary artery bypass grafting were excluded from the study. Twenty six patients had anterior infarction and 20 had inferior infarction.

The mean (SD) intervals between myocardial infarction and resting SPECT, exercise SPECT, and PET were 16 (9), 24 (8), and 21 (11) days, respectively. Three scintigraphic studies for each patient were performed within three weeks.

\section{RADIOPHARMACEUTICALS}

BMIPP, an iodine-123 labelled $\beta$ methyl branched fatty acid analogue, was prepared and supplied by Nihon Medi-Physics Co (Hyogo, Japan). Thallium-201 was obtained from commercial laboratories.

A small cyclotron (NKK Corporation, Kanagawa, Japan) was used for production of nitrogen-13 and fluorine-18. $\mathrm{NH}_{3}$ was produced by the method reported by Mulholland et al. ${ }^{18}$ FDG was synthesised by the method reported by Hamacher et al. ${ }^{19}$

RESTING BMIPP AND THALLIUM-201 DUAL ISOTOPE SPECT

After overnight fasting, each patient received an intravenous injection of BMIPP $(111 \mathrm{MBq})$ and thallium-201 (111 MBq) at rest. Initial images were obtained 20 minutes after the injection, and delayed images were obtained four hours later. SPECT was performed using a single head gamma scintillation camera equipped with a low energy, all purpose parallel hole collimator. Thirty two equidistant projections were acquired (30 s/projection) over $180^{\circ}$ from the right anterior oblique to the left posterior oblique. The images from the two energy windows $(159 \mathrm{keV} \pm 7.5 \%$ for iodine123 and $70 \mathrm{keV} \pm 10 \%$ for thallium-201) were collected in separate $64 \times 64$ matrices and then reconstructed using the Butterworth filter and Shepp and Rogan filter along the short axis, the horizontal long axis, and the vertical long axis of the heart. Images were normalised to the maximum count in each image set, and displayed as a colour scale image by a computer system (Scintipac-7000, Shimadzu Corporation, Kyoto, Japan). No downscatter correction was performed.

EXERCISE THALLIUM-201 SPECT

Each patient performed symptom limited exercise on a bicycle ergometer in the sitting position. Twelve lead electrocardiograms and blood pressure measurements were obtained at baseline and at every minute of exercise. The initial workload was 25 or 50 watts and was increased by 25 watts every two minutes until the end point of the exercise was reached. The end points included excessive fatigue, dyspnoea, dizziness, moderate to severe angina, hypotension, diagnostic ST depression $(>1.5$ $\mathrm{mm}$ horizontal or downsloping, or $>2.0 \mathrm{~mm}$ upsloping), or significant arrhythmia. At peak exercise, a dose of $111 \mathrm{MBq}$ thallium-201 was injected intravenously and the patient was encouraged to exercise for an additional minute. Initial images were obtained immediately after the termination of exercise and delayed images were obtained four hours later. SPECT was performed as in the resting study.

\section{PET IMAGING}

PET imaging was performed with a ShimadzuSET 1400 W-10 PET scanner (Headtome IV, Shimadzu Corporation). This can obtain seven slices simultaneously with a $13 \mathrm{~mm}$ interval, slice thickness of $11 \mathrm{~mm}$ FWHM, and spatial resolution of $4.5 \mathrm{~mm}$ FWHM. The axial, 6.5 $\mathrm{mm}$ interval, $\mathrm{Z}$ motion of the scanner every minute provided a total of 14 contiguous transverse slices of the myocardium. A 10 minute transmission scan was performed using a rotating germanium- 68 rod source. The acquired data were used to correct emission images for body attenuation. On completion of the attenuation scan, the patient remained in the supine position on the table and was injected intravenously with 259 to $740 \mathrm{MBq}$ of $\mathrm{NH}_{3}$. After a three minute delay to allow pulmonary background activity to clear, myocardial perfusion imaging was performed for 10 minutes.

After at least five hours of fasting and two to three hours after the perfusion scan, the patient received an intravenous injection of 148 to $407 \mathrm{MBq}$ of FDG. Sixty minutes were allowed for cardiac uptake of FDG. Imaging of glucose utilisation was then obtained for 10 minutes.

Images were collected in $256 \times 256$ matrices and then reconstructed using the Butterworth filter and the Ramp filter along the short axis, the horizontal long axis, and the vertical long axis of the heart by a computer system (Dr 


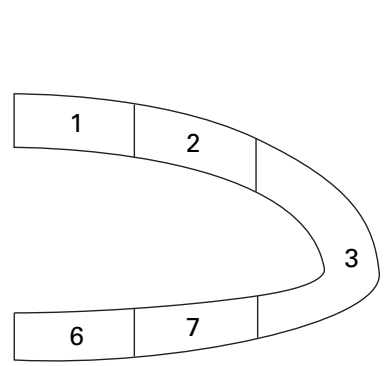

Vertical long axis view

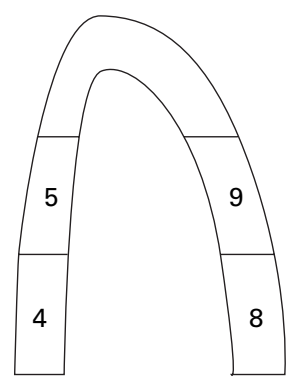

Horizontal long axis view
1 Basal-anterior

2 Mid-anterior

3 Apical

4 Basal-septal

5 Mid-septal

6 Basal-inferior

7 Mid-inferior

8 Basal-lateral

9 Mid-lateral

Figure 1 Schematic diagram of nine segments of left ventricle.
View, Asahi-Kasei Joho System Co, Tokyo, Japan). Images were normalised to the maximum count in each image set, and were displayed as a colour scale image.

DATA ANALYSIS

The SPECT and PET images were estimated visually. The left ventricular myocardium was divided into nine segments (basal anterior, mid-anterior, basal septal, mid-septal, basal inferior, mid-inferior, basal lateral, mid-lateral, apical) on a vertical long axis slice and a horizontal long axis slice, and a single segment representing the apex was assessed from the vertical long axis slice (fig 1). Anteroseptal-apical segments, inferior segments, and lateral segments were considered to be left anterior descending coronary artery territory, right coronary artery territory, and left circumflex coronary artery territory, respectively. SPECT and PET images were analysed individually by two experienced observers blinded to the patients' clinical data. Disagreements in interpretation were resolved by consensus of the two observers. BMIPP and thallium-201 defects in each segments were scored using a five point grading system $(0=$ normal, $1=$ mild defect, $2=$ moderate defect, $3=$ severe defect, and $4=$ no uptake).

Table 1 Thallium-201 redistribution pattern and defect score of the initial image on resting SPECT (number of segments)

\begin{tabular}{lrrrrr}
\hline & \multicolumn{5}{c}{ Defect score } \\
\cline { 2 - 6 } & 0 & \multicolumn{1}{c}{1} & \multicolumn{1}{c}{3} & \multicolumn{1}{c}{4} \\
\hline Fixed defect & 0 & 10 & 23 & 10 & 45 \\
Reverse redistribution & 5 & 14 & 10 & 11 & 0 \\
Redistribution & 0 & 0 & 0 & 4 & 4 \\
p Value & & & & & $<0.0001$ \\
\hline
\end{tabular}

Table 2 Relation between redistribution pattern of resting thallium-201 SPECT and myocardial metabolism (number of segments)

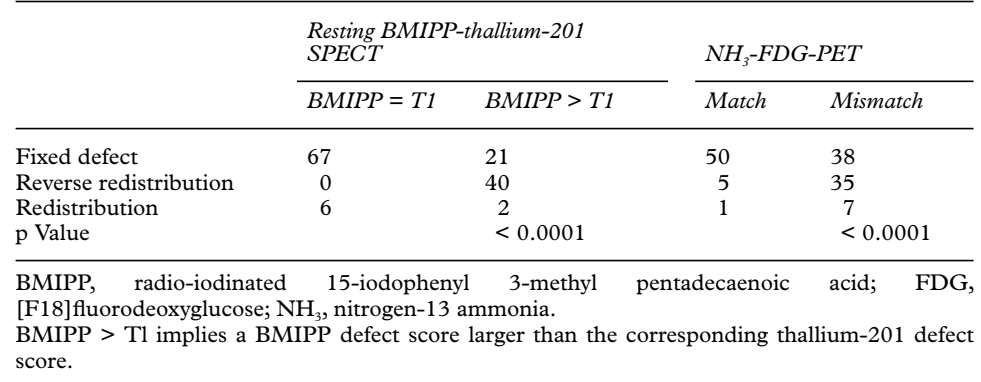

REDISTRIBUTION PATTERNS OF RESTING THALLIUM-201 SPECT

Relative to the defect score of the initial image, a decrease in defect score of the delayed image was defined as rest redistribution and an increase was defined as reverse redistribution. Fixed defect was defined as the defect of the delayed image equal to that of the initial image for scores not equal to zero.

COMPARISON OF RESTING BMIPP AND

\section{THALLIUM-201 IMAGES}

Comparison of defects on resting BMIPP and thallium-201 images was performed for each initial image. A segment was determined to be BMIPP > T1 when the defect score of the BMIPP image was larger than that of the thallium-201 image, which was considered to represent decreased fatty acid uptake compared with myocardial perfusion. Similarly, a segment was determined to be BMIPP $=\mathrm{T} 1$ when the defect scores of the BMIPP image and the thallium-201 image were equal, which was considered to represent matched fatty acid uptake and myocardial perfusion.

\section{COMPARISON OF RESTING AND EXERCISE} THALLIUM-201 SPECT

Many investigators report that many thallium201 defects on the delayed images from exercise study improve in the initial images from resting study, and that the latter reveal myocardial viability more precisely than the former. ${ }^{112}$ We have reported that the initial images reflect viability more precisely than delayed images in patients with reverse redistribution on resting thallium-201 scan. ${ }^{4}$ Thus we compared the delayed images from the exercise study and the initial images from the resting study to assess whether the redistribution on the delayed image from the exercise study was sufficient, or whether the defect on the delayed image from exercise study corresponded to myocardial viability.

Segments in the infarct related territory were analysed. A segment was determined to be positive for exercise induced ischaemia when the defect score of the initial image from the exercise study was larger than that of the initial image from the resting study. Similarly, a segment was determined to be negative for exercise induced ischaemia when the defect scores were equal. In the segments with exercise induced ischaemia, redistribution was determined to be rapid when the defect score of the delayed image from the exercise study was equal to that of the initial image from the resting study, and was defined as slow when the defect score of the delayed image from the exercise study was larger than that of the initial image from resting study.

COMPARISON OF $\mathrm{NH}_{3}$ AND FDG ON PET

$\mathrm{NH}_{3}$ and FDG images from corresponding segments were compared. Two experienced observers visually interpreted the uptake on both images. The uptake of FDG in noninfarcted myocardium is very low under fasting conditions. The relation between perfusion and metabolism was defined as mismatched when 
Table 3 Relation between the findings of exercise thallium-201 SPECT and myocardial metabolism (number of segments)

\begin{tabular}{|c|c|c|c|c|}
\hline & \multicolumn{2}{|c|}{$\begin{array}{l}\text { Resting BMIPP-thallium-201 } \\
\text { SPECT }\end{array}$} & \multicolumn{2}{|c|}{$\mathrm{NH}_{3}-\mathrm{FDG} \mathrm{PET}$} \\
\hline & $B M I P P=T 1$ & $B M I P P>T 1$ & Match & Mismatch \\
\hline \multicolumn{5}{|l|}{ Exercise induced ischaemia } \\
\hline Negative & 65 & 8 & 39 & 34 \\
\hline \multicolumn{5}{|l|}{ Positive } \\
\hline Rapid stress redistribution & 8 & 9 & 12 & 5 \\
\hline Slow stress redistribution & 5 & 49 & 12 & 42 \\
\hline $\mathrm{p}$ Value & & $<0.0005$ & & $<0.0001$ \\
\hline
\end{tabular}

BMIPP, radio-iodinated 15-iodophenyl 3-methyl pentadecaenoic acid; FDG, [F18]fluorodeoxyglucose; $\mathrm{NH}_{3}$, nitrogen-13 ammonia.

BMIPP $>$ Tl implies a BMIPP defect score larger than the corresponding thallium-201 defect score.

Table 4 Relation between the findings of resting and exercise thallium-201 SPECT (number of segments)

\begin{tabular}{lllll}
\hline & \multicolumn{3}{l}{ Resting } & thallium-201 SPECT \\
\cline { 2 - 5 } & $\begin{array}{l}\text { Fixed } \\
\text { defect }\end{array}$ & $\begin{array}{l}\text { Reverse } \\
\text { redistribution }\end{array}$ & Redistribution & Normal \\
\hline $\begin{array}{l}\text { Exercise thallium-201 SPECT } \\
\quad \begin{array}{l}\text { Negative } \\
\text { Positive }\end{array} \quad\end{array}$ & 61 & 2 & 6 & 4 \\
$\quad \begin{array}{l}\text { Rapid stress redistribution } \\
\quad \text { Slow stress redistribution }\end{array}$ & 9 & 2 & 0 & 6 \\
$\mathrm{p}$ Value & 4 & 36 & 2 & 2 \\
\hline
\end{tabular}

FDG uptake was definitely higher than both $\mathrm{NH}_{3}$ uptake of the segment and FDG uptake of non-infarcted segments (this was considered to represent increased glucose uptake compared with myocardial perfusion), and was defined as matched when FDG uptake was less than or similar to $\mathrm{NH}_{3}$ uptake of the segment (this was considered to represent matched glucose uptake compared with myocardial perfusion $\left.^{20}\right)$.

CORONARY ARTERIOGRAPHY AND LEFT VENTRICULOGRAPHY

Six patients underwent successful coronary angioplasty of the infarct related artery within 24 hours of the onset of myocardial infarction. Forty patients underwent coronary arteriography during the subacute phase but had no coronary intervention before the scintigraphic studies. Coronary arteriography was performed in multiple projections using standard techniques. Coronary narrowing was estimated by visual analysis and expressed as the maximum percent narrowing of luminal diameter. Estimates were determined by two experienced observers without access to the findings of the scintigraphic studies. Coronary narrowing exceeding $50 \%$ was considered significant. Forty one patients had single vessel disease and five had two vessel disease. Left ventriculography was performed in 40 patients. The mean ejection fraction of the left ventricle in these patients measured by a centre line method was $40(12) \%$.

\section{STATISTICS}

We used the $\chi^{2}$ test to assess the incidence of phenomena in the subgroups. Probability $(p)$ values $<0.05$ were considered significant.
Table 5 Relation between \% diameter stenosis of infarct related artery and redistribution pattern of resting thallium-201 SPECT (number of segments)

\begin{tabular}{lrrrc}
\hline & \multicolumn{3}{c}{ \% Diameter stenosis } \\
\cline { 2 - 5 } & $\leqslant 50$ & $\leqslant 90$ & 99 & 100 \\
\hline Fixed defect & 21 & 26 & 14 & 27 \\
Reverse redistribution & 18 & 15 & 6 & 1 \\
Redistribution & 1 & 2 & 1 & 4 \\
p Value & & & & $<0.01$ \\
\hline
\end{tabular}

\section{Results}

REVERSE REDISTRIBUTION OF THALLIUM-201 ON RESTING SPECT AND MYOCARDIAL METABOLISM Resting thallium-201 SPECT showed 88 segments with a fixed defect, 40 with reverse redistribution, and eight with redistribution in infarct related territories. The distribution of defect scores of the initial images on resting thallium-201 SPECT are shown in table 1. Defects with reverse redistribution contained more defects with small defect scores than those with fixed defects. The incidence of BMIPP > T1 and $\mathrm{NH}_{3}-\mathrm{FDG}$ mismatch was significantly higher in segments with reverse redistribution than in segments with a fixed defect (table 2). In the 57 segments with a defect score of 4 from the delayed images in resting SPECT, 10 of 12 segments (83\%) with reverse redistribution showed $\mathrm{NH}_{3}-\mathrm{FDG}$ mismatch, while only 16 of 45 segments $(36 \%)$ with a fixed defect showed $\mathrm{NH}_{3}-\mathrm{FDG}$ mismatch $(p<0.01)$.

SLOW STRESS REDISTRIBUTION OF THALLIUM-201 ON DELAYED IMAGE FROM EXERCISE SPECT AND MYOCARDIAL METABOLISM

In 144 segments of infarct related territories, defect scores of initial image of exercise thallium-201 SPECT were equal to or greater than 1 . Of these segments, 73 were negative for exercise induced ischaemia. Of the 71 segments with exercise induced ischaemia, 17 showed rapid stress redistribution on delayed image from exercise SPECT and 54 showed slow stress redistribution. The incidence of BMIPP > T1 and $\mathrm{NH}_{3}-\mathrm{FDG}$ mismatch was significantly higher in segments with slow stress redistribution than in segments with rapid stress redistribution or in segments without exercise induced ischaemia (table 3 ).

RELATION BETWEEN EXERCISE AND RESTING THALLIUM-201 SPECT

In 73 segments without exercise induced ischaemia, only two (3\%) showed reverse redistribution on resting thallium-201 SPECT. In 17 segments with exercise induced ischaemia and rapid stress redistribution, only two $(12 \%)$ showed reverse redistribution on resting thallium-201 SPECT, but in 54 segments with exercise induced ischaemia and slow stress redistribution, $36(67 \%)$ showed reverse redistribution (table 4, fig 2).

SEVERITY OF STENOSIS OF INFARCT RELATED ARTERY AND KINETICS OF THALLIUM-201 Segments with reverse redistribution were supplied by fewer stenotic arteries than segments with fixed defect on resting thallium- 


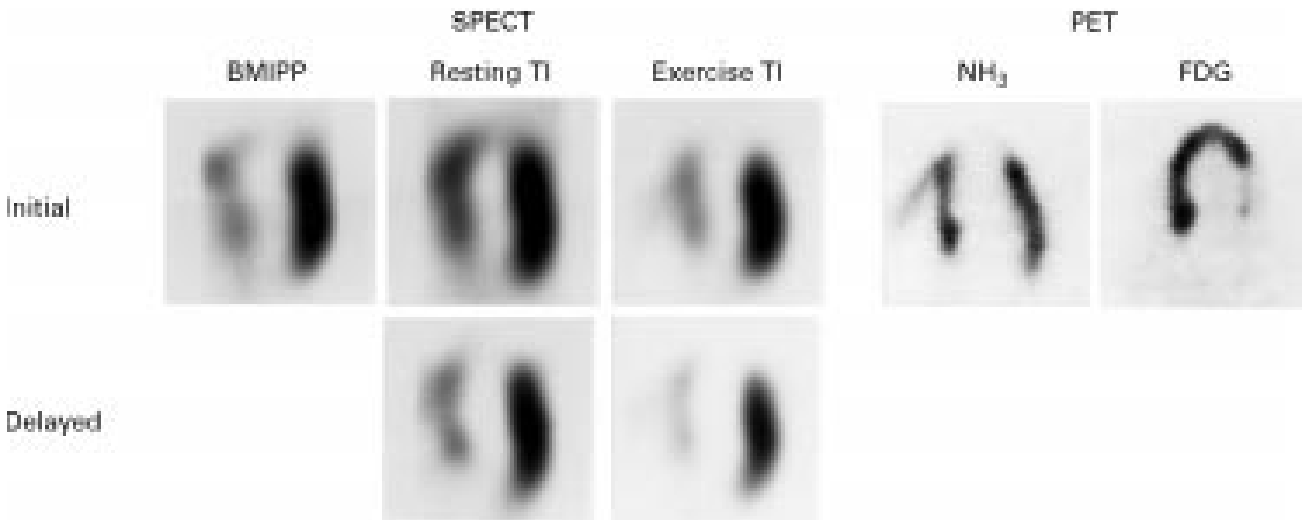

Figure 2 A representative case of anteroseptal-apical myocardial infarction (horizontal long axis view). The initial image of BMIPP SPECT shows a moderate defect in the septum and no uptake in the apex. The initial image of the resting thallium-201 SPECT shows a mild defect in the septum and a moderate defect in the apex, and the delayed image of the resting thallium-201 SPECT shows a moderate defect in the septum and no uptake in the apex. Both of the initial and delayed images of exercise thallium-201 SPECT show severe defects in the septum and no uptake in the apex. The NH PET image shows a mild defect in the septum and no uptake in the apex. The FDG PET image shows increased uptake in the septum and apex. Septum and apex have resting reverse redistribution, exercise induced ischemia, and slow stress redistribution on thallium-201 SPECT. These segments show less BMIPP uptake and more FDG uptake than uptake of the perfusion tracers.

201 SPECT (table 5). Four of eight segments with rest redistribution had collateral circulation. Segments with exercise induced ischaemia and slow stress redistribution were supplied by arteries that were less stenotic than arteries supplying segments without exercise induced ischaemia, or segments with exercise induced ischaemia and rapid stress redistribution (table 6).

\section{Discussion}

Our study shows that the incidence of discordant uptake of BMIPP (that is, uptake less than that of thallium-201) and discordant uptake of FDG (that is, more than that of $\mathrm{NH}_{3}$ ) was significantly higher in myocardial segments with reverse redistribution of thallium-201 in resting SPECT or with slow stress redistribution of thallium-201 on delayed images from exercise SPECT. We are not aware of any previous reports investigating both fatty acid and glucose metabolism in the myocardium that show reverse redistribution of thallium-201 on resting SPECT, or slow stress redistribution of thallium-201 on four hour delayed images from exercise SPECT in patients with acute myocardial infarction. In addition, $83 \%$ of myocardial segments with reverse redistribution and no thallium-201 uptake on delayed images in resting SPECT had $\mathrm{NH}_{3}-\mathrm{FDG}$ mismatch, indicating ischaemic but viable myocardium.

Table 6 Relation between \% diameter stenosis of infarct related artery and the findings on exercise thallium-201 SPECT (number of segments)

\begin{tabular}{lllll}
\hline & \multicolumn{3}{l}{$\%$ Diameter stenosis } \\
\cline { 2 - 5 } & $\leqslant 50$ & $\leqslant 90$ & 99 & 100 \\
\hline Exercise thallium-201 SPECT & 17 & 20 & 10 & 26 \\
Exercise induced ischaemia & 3 & 7 & 4 & 3 \\
$\quad$ Negative & 22 & 20 & 7 & 5 \\
$\quad \begin{array}{l}\text { Positive } \\
\quad \text { Rapid stress redistribution }\end{array} \quad$ & & & & $<0.05$ \\
$\quad$ Slow stress redistribution & & & & \\
\hline
\end{tabular}

RELATION BETWEEN THE KINETICS OF THALLIUM-201 AND MYOCARDIAL METABOLISM Soufer et al reported that $72 \%$ of myocardial segments with reverse redistribution in stress planar thallium-201 scintigrams in patients with chronic coronary artery disease showed $\mathrm{NH}_{3}-\mathrm{FDG}$ mismatch. ${ }^{21}$ Marin-Neto et al studied 39 patients with chronic stable coronary artery disease and reverse redistribution of thallium-201 on exercise-redistribution SPECT; they reported that in 16 patients assessed by PET, all regions with reverse redistribution had viable myocardium. ${ }^{22}$ The finding of reverse redistribution on exercise thallium-201 SPECT is consistent with the finding of reverse redistribution on resting thallium-201 SPECT in our study.

Matsunari et al have investigated the relation between myocardial fatty acid metabolism and new fill-in after thallium-201 reinjection in patients with chronic coronary artery disease and persistent defects on standard exercise redistribution thallium-201 imaging. ${ }^{17}$ BMIPP uptake was found to be less than thallium-201 uptake on reinjection imaging in $82 \%$ of myocardial segments with new fill-in after reinjection, but only $19 \%$ of segments with no fill-in discordantly decreased BMIPP uptake. Thallium-201 reinjection imaging was used to assess resting myocardial perfusion and for comparison with resting BMIPP imaging, but the reinjection image is not strictly comparable with the resting perfusion image. In contrast, we used resting BMIPP and thallium-201 dual isotope SPECT in our study, and this allowed precise comparison of fatty acid uptake and resting perfusion.

In patients with chronic coronary artery disease the incidence of $\mathrm{NH}_{3}-\mathrm{FDG}$ mismatch has been found to be significantly higher in myocardial segments with new fill-in after thallium-201 reinjection than in segments with redistribution on delayed images. ${ }^{23}$ These findings are consistent with the results of the present study in segments with slow or rapid 
stress redistribution on delayed image from exercise SPECT.

RELATION BETWEEN RESTING REVERSE

REDISTRIBUTION AND SLOW STRESS

REDISTRIBUTION

The time required to complete thallium-201 redistribution following stress has been reported to be related to the severity of stenosis in the coronary artery supplying the area concerned. ${ }^{24}$ However, in the present study segments with exercise induced ischaemia and slow stress redistribution in exercise SPECT were supplied by less stenotic arteries than segments with exercise induced ischaemia and rapid stress redistribution. Also, the segments with reverse redistribution in resting SPECT were supplied by less stenotic arteries than segments with a fixed defect. In acutely infarcted myocardium, conditions other than myocardial regional blood flow (that is, uptake and washout rate of thallium-201 in the myocardium) may play a part in the reverse redistribution during resting scintigraphy and the slow stress redistribution during exercise study.

Weiss et al have reported that all acute myocardial infarcts with reverse redistribution in resting scintigraphy had patent infarct related coronary arteries. ${ }^{3}$ However, Hecht et al have reported that $85 \%$ of patients with reverse redistribution during stress scintigraphy have a stenosed coronary artery ( $\geqslant 90 \%$ stenosis).$^{25} \mathrm{It}$ has also been reported that reverse redistribution does not correlate closely with the degree of coronary artery disease. ${ }^{46}$ These differences among the previous and the present studies are thought to be caused by the differences of patient population, the time of the test, and the definition of reverse redistribution of each report.

The results of our study indicate that in myocardial segments with reverse redistribution on resting SPECT or slow stress redistribution on exercise SPECT, the major energy source changed from fatty acids to glucose, perhaps leading to a decrease in ATP production. Reverse redistribution on resting thallium-201 SPECT was reported in viable myocardium with severe contraction abnormality in patients with acute myocardial infarction. ${ }^{4}$ Also, a strong correlation has been observed between the degree of reverse redistribution on resting thallium-201 SPECT and the degree of insufficiency of redistribution on delayed images from exercise thallium-201 SPECT. ${ }^{5}$ The correlation between reverse redistribution on resting SPECT and slow stress redistribution on delayed images from exercise SPECT was also observed in the present study. Accordingly, we thought that the phenomena of reverse redistribution in resting SPECT and slow stress redistribution in exercise SPECT might have a common mechanism related to the metabolic abnormality of the myocardium.

\section{STUDY LIMITATIONS}

The patient selection in our study was performed retrospectively, and was biased. As a result, most of the patients had single vessel disease. We also assessed the SPECT and PET images visually. To confirm our results, quantitative analysis in a larger patient population should be performed, including many patients with multivessel disease.

We did not investigate the relation between thallium-201 kinetics and the left ventricular wall motion abnormality or its serial changes, which would be necessary to confirm whether decreased BMIPP uptake and increased FDG uptake under fasting conditions indicates potential reversibility of the wall motion abnormalities after revascularisation.

\section{CONCLUSIONS}

Our results suggest that thallium-201 kinetics that indicate the presence of reverse redistribution on resting SPECT or slow stress redistribution on exercise SPECT are strongly correlated with abnormality of myocardial metabolism, as indicated by a decrease in fatty acid uptake and an increase in glucose uptake. Our study shows that thallium-201 myocardial scintigraphy provides information not only about myocardial perfusion and viability but also about myocardial metabolism in patients with acute myocardial infarction. We greatly appreciate the secretarial assistance of Ms Harumi
Baba and Ms Ayako Kobayashi. We thank the technologists of Baba and Ms Ayako Kobayashi. We thank the technologist
Division of Nuclear Medicine for their technical assistance.

1 Pohost GM, Zir LM, Moore RH, et al. Differentiation of transiently ischemic from infarcted myocardium by serial imaging after a single dose of thallium-201. Circulation 1977;55:294-302.

2 Maddahi J, Berman DS. Reverse redistribution of thallium201. F Nucl Med 1995;36:1019-21.

3 Weiss AT, Maddahi J, Lew AS, et al. . Reverse redistribution of thallium-201: a sign of nontransmural myocardial infarction with patency of the infarct-related coronary artery. $\mathcal{F}$ Am Coll Cardiol 1986;7:61-7.

4 Yamagishi $\mathrm{H}$, Itagane $\mathrm{H}$, Akioka $\mathrm{K}$, et al. Clinical significance of reverse redistribution on thallium-201 single photon emission computed tomography in patients with acute myocardial infarction. fpn Circ f 1992;56:1095-105.

5 Yamagishi H, Akioka K, Ipn Circ f 1992;56:1095-105. Yamagishi $\mathrm{H}$, Akioka $\mathrm{K}$, Itagane $\mathrm{H}$, et al. Relationship between insufficient redistribution in exercise thallium-201 myocardial single-photon emission computed tomography
and reverse redistribution at rest. fpn Circ f 1995;59:23and 32 .

6 Brunken RC, Kotou S, Nienaber CA, et al. PET detection of viable tissue in myocardial segments with persistent defects at T1-201 SPECT. Radiology 1989;172:65-73.

7 Cloninger KG, DePuey EG, Garcia EV, et al. Incomplete redistribution in delayed thallium-201 single photon emission computed tomography (SPECT) images: an overestimation of myocardial scarring. $\mathcal{F}$ Am Coll Cardiol 1988;12: 955-63.

8 Kiat H, Berman DS, Maddahi J, et al. Late reversibility of tomographic myocardial thallium-201 defects: an accurate 1456-63.

9 Rocco TP, Dilsizian V, McKusick KA, et al. Comparison of thallium redistribution with rest "reinjection" imaging for the detection of viable myocardium. Am f Cardiol 1990;66: 158-63.

10 Dilszian V, Rocco TP, Freedman NMT, et al. Enhanced detection of ischemic but viable myocardium by the
reinjection of thallium after stress redistribution imaging. $N$ reinjection of thallium after stes

11 Blood DK, McCarthy DM, Sciacca RR, et al. Comparison of single-dose and double-dose thallium-201 myocardial perfusion scintigraphy for the detection of coronary artery disease and prior myocardial infarction. Circulation 1978; 58:777-88.

12 Ritchie JL, Albro PC, Caldweel JH, et al. Thallium-201 myocardial imaging: a comparison of the redistribution and rest images. $\mathcal{F ~ N u c l ~ M e d ~ 1 9 7 9 ; 2 0 : 4 7 7 - 8 3 . ~}$

13 Goodman MM, Kirsch G, Knapp FF. Synthesis and evaluation of radioiodinated terminal p-iodo-phenylsubstituted - and -methylbranched fatty acids. F Med Chem 1984;27:390-7.

14 Knapp FF, Ambrose KR, Goodman MM. New radioiodinated methyl-branched fatty acids for cardiac studies. Eur $\mathcal{F}$ Nucl Med 1986;12:S39-44.

15 Marshall M, Tillisch JH, Phelps ME, et al. Identification and differentiation of resting myocardial ischemia and infarction in man with positron computed tomography, 
${ }^{18} \mathrm{~F}$-labeled fluorodeoxyglucose and N-13 ammonia. Circulation 1983;67:766-78

16 Tillisch J, Brunken R, Marshall R, et al. Reversibility of cardiac wall-motion abnormalities predicted by positron tomography. N Engl f Med 1986;314:884-8.

17 Matsunari I, Fujino S, Taki J, et al. Impaired fatty acid uptake in ischemic but viable myocardium identified by

thallium-201 reinjection. Am Heart $\mathcal{F}$ 1996;131:458-65.
8 Mulholland GK, Kilbourn MR, Moskwa JJ. Direct simulta-

neous production of $\left[{ }^{15} \mathrm{O}\right]$ water and $\left[{ }^{13} \mathrm{~N}\right]$ ammonia or $\left[{ }^{18} \mathrm{~F}\right]$ fluoride ion by $26 \mathrm{MeV}$ proton irradiation of a double chamber water target. Appl Radiat Isot 1990;41:1193-9.

19 Hamacher K, Coenen HH, Stocklin G. Efficient stereospecific synthesis of no-carrier-added 2-[18F]-fluoro-2-deoxyD-glucose using aminopolyether supported nucleophilic substitution. F Nucl Med 1986;27:235-8.

20 Tamaki N, Yonekura Y, Yamashita K, et al. Positron emission tomography using fluorine-18 deoxyglucose in evaluating of coraphy using fluorine-18 deoxyglucose in 1989;64:860-5.

21 Soufer R, Dey HM, Lawson AJ, et al. Relationship between reverse redistribution on planar thallium scintigraphy and regional myocardial viability: a correlative PET study. 7 Nucl Med 1995;36:180-7.

22 Marin-Neto JA, Dilsizian V, Arrighi JA, et al. Thallium reinjection demonstrates viable myocardium in regions with reverse redistribution. Circulation 1993;88:1736-45.

23 Tamaki N, Ohtani H, Yamashita K, et al. Metabolic activity in the areas of new fill-in after thallium-201 reinjection: comparison with positron emission tomography using fluorine-18-deoxyglucose. F Nucl Med 1991;32:673-8.

24 Gutman J, Berman DS, Freeman $M$, et al. Time to completed redistribution of thallium-201 in exercise myocardial scintigraphy: relationship to the degree of coronary artery stenosis. Am Heart $\mathcal{F}$ 1983;106:989-95.

25 Hecht HS, Hopkins JM, Rose JG, et al. Revers redistribution: worsening of thallium-201 myocardial images from exercise to redistribution. Radiology 1981;140: 177-81.

26 Silberstein EB, DeVries DF. Reverse redistribution phenomenon in thallium-201 stress tests: angiographic correnomenon in thallium-201 stress tests: angiographic corre-
lation and clinical significance. 7 Nucl Med 1985;26:70710. 